\title{
Oxidative Stress and Antioxidant Vitamins Status of Diabetic Subjects Supplemented with Antioxidant Rich Supplement (Alphabetic) Attending Usmanu Danfodiyo University Teaching Hospital, Sokoto, Nigeria
}

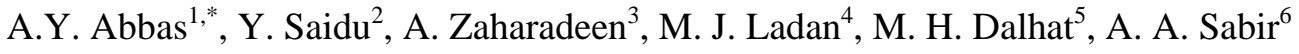 \\ ${ }^{1}$ Department of Biochemistry, Faculty of Science, Usmanu Danfodiyo University \\ Sokoto, Nigeria \\ Email: ayabbas2002 [AT] yahoo.com \\ ${ }^{2}$ Department of Biochemistry, Faculty of Science, Usmanu Danfodiyo University \\ Sokoto, Nigeria \\ Email: yusdab [AT] yahoo.com \\ ${ }^{3}$ Department of Biochemistry, Faculty of Science, Usmanu Danfodiyo University \\ Sokoto, Nigeria \\ Email: Zaharadeen1988 [AT] gmail.com \\ ${ }^{4}$ Department of Biochemistry, Faculty of Science, Usmanu Danfodiyo University \\ Sokoto, Nigeria \\ Email: dladanjangebe [AT] yahoo.com \\ ${ }^{5}$ Department of Biochemistry, Faculty of Science, Usmanu Danfodiyo University \\ Sokoto, Nigeria \\ Email: mahmooddalhat [AT] gmail.com \\ ${ }^{6}$ Department of Medicine, College of Health sciences, Usmanu Danfodiyo University \\ Sokoto, Nigeria \\ Email: ansabirl [AT] yahoo.com \\ *Corresponding author: A.Y. Abbas
}

\begin{abstract}
Diabetes mellitus is a group of metabolic diseases characterized by hyperglycemia resulting from defects in insulin secretion / insulin action or both which could leads to increase level of oxidative stress and decrease in antioxidant defense system and may play role in the development of diabetic complications. The present study examines the effects of antioxidant rich supplements (Alphabetic) on antioxidant status of one hundred (100) diabetic subjects attending Usmanu Danfodiyo University Teaching Hospital (UDUTH) for at least one year and the results were compared with those of fifty (50) diabetes mellitus non-supplemented and fifty (50) apparently healthy non-diabetic subjects. Standard analytical procedures were used for all the parameters measured including the effects of supplementation on Fasting Blood Glucose, oxidative stress marker (MDA), Reduced gluthathione and antioxidant vitamins $(C, E$ and $\beta$-carotene) parameters were evaluated. The results revealed that the supplementation significantly $(P<0.05)$ reduce Serum glucose and Malondialdehyde and also significantly $(P<0.05)$ increases Vitamin $C$, Vitamin $E$ and Reduced glutathione compared with the non-supplement group. The findings suggests that supplementation with Alphabetic might reduce the risk of oxidative stress associated with diabetes mellitus leading to diabetic complications and might be beneficial in the routine treatment of diabetes mellitus.
\end{abstract}

Keywords--- Antioxidant, Diabetic, Malondialdehyde, hyperglycemia, Alphabetic

\section{INTRODUCTION}

Diabetes mellitus (DM) is a group of metabolic diseases characterized by persistent hyperglycemia and disturbances in the metabolism of fuel compounds as a result of absolute or relative deficiency in insulin secretion or/and insulin action [1].Chronic hyperglycemia is associated with long-term damage, dysfunction and failure of normal functioning of various organs, especially the eyes, kidneys, nerves, heart, and blood vessels [2][3]. Diabetes-specific microvascular diseases are the leading cause of blindness, renal failure, and nerve damage [4].The global burden of diabetes mellitus has risen 
dramatically over the last two decades and diabetes mellitus is expected to affect more than 500 million adults worldwide by 2030 [5]. The estimated worldwide prevalence of diabetes mellitus among adults in 2010 was (6.4\%) around 285 million people [6]. In Nigeria, the prevalence of diabetes mellitus is about $2.7 \%$ [7]. The adoption of a sedentary lifestyle, the consumption of non-traditional foods and a genetic predisposition to the disease are thought to be the major risk factors of the epidemic [8][9]. Presently, the disorder has no known cure but could be controlled by drugs [10]. Strict glycemic control is the primary method of reducing the development and progression of microvascular complications. Also, aggressive treatment of dyslipidemia and hypertension decreases macrovascular complications [11]. Regular supplementation with antioxidant rich supplements or nutraceuticals can also used to treats diabetes mellitus [12].Oxidative Stress (OS) is a general term used to describe the steady state level of oxidative damage in a cell, tissue, or organ, caused by the reactive oxygen species (ROS) [13]. Reactive oxygen species, such as free radicals and peroxides, represent a class of molecules that are derived from the metabolism of oxygen and exist inherently in all aerobic organisms.ROS consist of oxygen free radicals such as superoxide anion $\left(\mathrm{O}_{2}^{-}\right)$, hydrogen peroxide $\left(\mathrm{H}_{2} \mathrm{O}_{2}\right)$, hydroxyl radical $\left(\mathrm{OH}^{-}\right)$, singlet oxygen, nitric oxide, and peroxynitrite [13]. Most of these free radicals are produced at low concentrations during normal physiological conditions in the body and are scavenged by endogenous enzymatic and non-enzymatic antioxidant systems that include superoxide dismutase, glutathione peroxidase, catalase, and vitamins ( $\mathrm{C}$ and $\mathrm{E}$ ). The over production of ROS usually induce damage to Proteins, lipids and DNA molecules [14]. Oxidative stress occurs when the production of reactive oxygen is greater than the body's ability to detoxify the reactive intermediates [15]. This imbalance leads to oxidative damage to proteins, lipids and DNA within the body. Since the body is incapable of keeping up with the detoxification of the free radicals, the damage continues to spread [16].Alphabetic (Antioxidant Formula) is the multi-vitamin / mineral supplement recommended by health professionals for people with diabetes. In addition to Lutein, chromium and 20 other diabetes specific nutrients, Alpha Betic contains something unique - a naturally occurring co-enzyme called Alpha Lipoic Acid Antioxidants have been indicated to boost the antioxidants defences, curtail the deleterious effects of reactive oxygen specie [12]. Therefore supplementation with antioxidant rich supplements should reverse the oxidative stress

\section{MATERIALS AND METHOD}

\subsection{Chemicals and Reagents}

All chemicals and reagents used for this study are of analytical grades.

\subsection{Ethical Approval}

Approval were obtained from Usmanu Danfodio University Teaching Hospital (UDUTH) ethical committe. The participants were given a consent form, with explanation about the purpose of the study. Consent forms were duly signed, by two participants.

\subsection{Sampling}

A total of 200 subjects were recruited for the study. These consisted of 50 Non-supplemented diabetic subjects attending the diabetic clinic of Usmanu Danfoyo University Teaching Hospital Sokoto, 100 diabetic subjects supplemented with antioxidant rich supplement (Alphabetic) attending the diabetic clinic of Usmanu Danfoyo University Teaching Hospital Sokoto and 50 apperently healthy subjects as Non-diabetic subjects. The non-diabetic subject were recruited from the general staff and students of UDUTH Sokoto, Nigeria.

\subsection{Sample Size Estimation}

The number of subjects for inclusion into the study was determined using the sample size formula for estimating sample size for descriptive studies [17].

$\mathrm{n}=\mathrm{Z}^{2} \mathrm{pq} / \mathrm{d}^{2}$

\section{Where}

$\mathrm{n}=$ Minimum sample size

$\mathrm{Z}=$ Standard normal distribution at confident interval (1.96)

$\mathrm{p}=$ prevalence obtained from previous study, i.e. prevelance of diabetes mellitus in Nigeria is put at $3.9 \%$ as at 2010 (Shaw et al., 2010)

$$
\begin{aligned}
& q=1-p \\
& d=\text { degree of confidence at } 5 \%=0.05 \\
& q=1-p=1-0.016=0.984
\end{aligned}
$$


$\mathrm{n}=(1.96)^{2}(0.039)(0.961)=58$

$(0.05)^{2}$

Therefore the minimum required sample is 60 .

For convenient sampling, we use 1:2:1. 50 Diabetic non-supplemented (DMNS), 100 Diabetic supplemented with Alphabetic (DMSA) and 50 Non-diabetic Mellitus (NODM)

\subsection{Biochemical Assays}

Blood samples of $5 \mathrm{mls}$ were collected in plane containers. After collection, samples were centrifuged at $4000 \mathrm{rpm}$ for 5 minutes, and serum were separated and stored in a deep freeze at $-20^{\circ} \mathrm{C}$.Serum glucose was estimated using the glucose oxidase method [18], $\beta$-carotene level was determined by the method of Pett [19], Vitamin C level was determined by the method of Baker and Frank [20], Vitamin E was determined by the method of Hashim and Schuttinger [21], Reduced glutathione level was estimated by the method Petterson and lazarow [22], Lipid Peroxidation (MDA) was estimated by the method of Shah and Walker [23]

\subsection{Statistical Analysis}

The data collected were express as mean \pm standard error of the mean. The significance of the differences between groups were determined using one way analysis of variance (ANOVA) and Duncan multiple test were used for comparison, $\mathrm{P}<0.05$ were considered as significant.

\section{RESULTS}

Table 1 showed the results of serum fasting blood sugar, reduced glutathione and malondialdehyde levels in Diabetes Mellitus Non-Supplemented, Diabetes Mellitus Supplemented with Alphabetic and Non-Diabetes Mellitus subjects.Serum fasting blood sugar levels in supplemented diabetic subjects shows significant $(\mathrm{P}<0.05)$ decrease when compared to non-supplemented diabetic subjects also there significant $(\mathrm{P}<0.05)$ decrease in fasting blood sugar in nondiabetic subjects compared to non-supplemented diabetic subjects. There is significant $(\mathrm{P}<0.05)$ decrease of reduced glutathione in non-supplemented diabetic subjects when compared to supplemented diabetic subjects and also reduced glutathione level shows significant $(\mathrm{P}<0.05)$ increase in non-diabetic subjects compared to diabetic non-supplemented. Malondialdehyde levels of diabetic subjects non- supplemented showed significant $(\mathrm{P}<0.05)$ increase compared to nondiabetic subjects but after supplementation with Alphabetic shows significant $(\mathrm{P}<0.05)$ decrease compared to diabetic nonsupplemented

Table 1: Serum Fasting Blood Sugar, Reduced Glutathione and Malonaldehyde Levels in Diabetes Mellitus and Non-Diabetes Mellitus subjects

\begin{tabular}{llll}
\hline & FBS $(\mathrm{mmol} / \mathrm{L})$ & GSH $(\mathrm{mg} / \mathrm{dL})$ & MDA $(\mathrm{mmol} / \mathrm{L})$ \\
\hline DMNS $(\mathrm{n}=50)$ & $13.75 \pm 1.43^{\mathrm{b}}$ & $95.29 \pm 15.29^{\mathrm{a}}$ & $41.23 \pm 3.93^{\mathrm{b}}$ \\
DMSA $(\mathrm{n}=100)$ & $7.06 \pm 0.36^{\mathrm{c}}$ & $197.81 \pm 7.09^{\mathrm{b}}$ & $20.07 \pm 3.41^{\mathrm{a}}$ \\
NODM $(\mathrm{n}=50)$ & $3.91 \pm 0.12^{\mathrm{a}}$ & $184.56 \pm 18.44^{\mathrm{b}}$ & $19.74 \pm 4.78^{\mathrm{a}}$ \\
\hline
\end{tabular}

Values are Mean \pm Standard Error of the Mean. Values bearing different superscripts on the same column differ significantly $(\mathrm{p}<0.05)$. FBS-fasting blood sugar, GSH-reduced glutathione. MDA-malonaldehyde, n- number of subjects, DMNS - Diabetic non-supplemented, DMSA- Diabetic supplemented with Alphabetic and NODM - Non-diabetic Mellitus.

Table 2 showed the results of serum antioxidant vitamins level in Diabetes Mellitus Non-Supplemented, Diabetes Mellitus Supplemented with Alphabetic and Non-Diabetes Mellitus subjects. The results reveal that, there is no significant $(\mathrm{P}<0.05)$ difference in vitamin $\mathrm{E}$ levels of non-supplemented diabetic subjects and non-diabetic subjects. Consequently, supplementation with Alphabetic, the supplemented diabetic subjects showed significant $(\mathrm{P}<0.05)$ increase in vitamin $\mathrm{E}$ levels compared to diabetic non-supplemented and non-diabetic subjects which are five (5) times than the non-diabetic subjects and fourteen (14) times then the diabetic non-supplemented subjects. A serum level of $\beta$-carotene shows significant $(\mathrm{P}<0.05)$ increase in supplemented diabetic subject compared to non- diabetic subjects and also there is significant $(\mathrm{P}<$ $0.05)$ increase in non-diabetic subjects compared to diabetic non-supplemented subjects. Serum levels of vitamin $C$ showed no significant $(\mathrm{P}<0.05)$ difference between non-diabetic subjects and non-supplemented diabetics subject but its higher in non-diabetic than diabetic non-supplemented, also there is no significant $(\mathrm{P}<0.05)$ difference in vitamin $\mathrm{C}$ levels between supplemented diabetic subjects and non-diabetic subjects but its higher in supplemented diabetic subjects than non-diabetic 
subjects. There is significant $(\mathrm{P}<0.05)$ improvement of vitamin $\mathrm{C}$ levels in supplemented diabetic subjects compared to non-supplemented diabetic subjects afer supplementation with Alphabetic.

Table 2: Serum Antioxidant Vitamins Level in Diabetes Mellitus Non-Supplemented, Diabetes Mellitus Supplemented with Alphabetic and Non-Diabetes Mellitus subjects

\begin{tabular}{lccc}
\hline & $\begin{array}{c}\text { Vitamin E } \\
(\mu \mathrm{mol} / \mathrm{L})\end{array}$ & $\begin{array}{c}\text { Vitamin C } \\
(\mu \mathrm{mol} / \mathrm{L})\end{array}$ & $\begin{array}{c}\beta-C a r o t e n e \\
(\mu \mathrm{mol} / \mathrm{L})\end{array}$ \\
\hline DMNS $(\mathrm{n}=50)$ & $14.18 \pm 1.38^{\mathrm{b}}$ & $232.79 \pm 23.17^{\mathrm{a}}$ & $1.92 \pm 0.34^{\mathrm{c}}$ \\
DMSA $(\mathrm{n}=100)$ & $194.90 \pm 31.86^{\mathrm{a}}$ & $789.12 \pm 133.2^{\mathrm{b}}$ & $3.72 \pm 1.32^{\mathrm{b}}$ \\
NODM $(\mathrm{n}=50)$ & $39.28 \pm 13.47^{\mathrm{b}}$ & $520.83 \pm 82.78^{\mathrm{c}}$ & $8.75 \pm 4.99^{\mathrm{a}}$ \\
\hline
\end{tabular}

Values are Mean \pm Standard Error of the mean. Values bearing different superscripts on the same column differ significantly $(\mathrm{p}<0.05)$. n- Number of subjects, DMNS - Diabetic non-supplemented, DMSA- Diabetic supplemented with Alphabetic and NODM - Non-diabetic Mellitus.

Table 3 showed the results of correlation Coefficient (r) of serum glucose with Serum antioxidant vitamins and oxidative stress marker (MDA) of Diabetic-supplemented with Alphabetic and Diabetics non-supplemented subjects. All the parameter, showed a negetive linear Correlation $(\mathrm{P}<0.05)$ while only MDA showed a positive linear Correlation to glucose. Negetive correlation means that as the level of glucose increase the levels of antioxidant vitamins and antioxidant enzymes goes down while positive correlation means that as the level of glucose increases the level of oxidative stress marker (MDA) also increases.

Table 3: Correlation Coefficient (r) of Serum Glucose with Serum Antioxidant Vitamins and Oxidative Stress Markers of Diabetics-supplemented with Alphabetic and Diabetics non-supplemented Subjects

\begin{tabular}{lll}
\hline Independent variable & $\begin{array}{l}\text { Diabeticsupplementedwith } \\
\text { Alphabetic (r) }\end{array}$ & Diabetic non-supplemented (r) \\
\hline$\beta$-carotene & -0.39 & -0.24 \\
Vitamin C & -0.16 & -0.08 \\
Vitamin E & -0.22 & -0.05 \\
GSH & -0.27 & -0.33 \\
MDA & 0.48 & 0.36 \\
\hline
\end{tabular}

Negetive value showed Negetive correlation, Positive value showed Positive correlation while Zero value shows there is no correlation between values. All the serum antioxidant vitamins and antioxidant enzymes are negatively correlated with serum glucose levels of both the diabetics supplemented and diabetic non-supplemented subjects. Oxidative stress marker shows a positive correlation $(\mathrm{P}<0.05)$ with serum glucose of both diabetic non-supplemented and diabetic supplemented with Alphabetic.

\section{DISCUSSION}

Diabetes mellitus is characterized by increased level of oxidative stress indices, decreased level of antioxidants defences and lipid abnormalities due to lipid peroxidation [24]. Generally, prolonged uncontrolled high blood glucose has been shown to results in elevated levels of serum glucose and oxidative stress indices as well as decreased levels of antioxidants defences and lipid abnormalities due to lipid peroxidation [24]. Supplementation with antioxidant rich supplements are though to be effective in increasing the activities of antioxidant defence enzymes, scavenging free radicals, preventing oxidative damage and thereby sparing lipid components of the cells against lipid peroxidation [25]. Deficiency of micronutrients and antioxidants in diabetes mellitus have been extensively described in many laboratories, and there is good evidence to suggest that oxidative stress may play a role in the pathogenesis of both type 2 diabetes mellitus [26].

The present study, revealed significant increase in fasting blood sugar $(\mathrm{P}<0.05)$ in diabetic non-supplemented subjects compared with the non-diabetic (Table 1). This findings are in accordance with [27], who observed a significantly $(\mathrm{P}<0.05)$ increase fasting blood sugar in diabetic subjects than normal subjects. There was also significantly $(\mathrm{P}<0.05)$ decrease level of fasting blood sugar in diabetic supplemented with Alphabetic compared with non-supplemented diabetic subjects (Table 1). This may be connected with increased availability of antioxidants that are important components and co-factors of the antioxidant enzymes [28]. In addition, some constituents of Alphabetic such as the antioxidant components, minerals, vitamins etc; could contribute to its antihyperglycemic effects by ameliorating the oxidative stress caused by diabetes. This is by inhibiting the formation of lipid peroxides, reactivation of the antioxidant enzymes, and restores the levels of reduced glutathione (GSH) and some mineral elements [29]. Reduced glutathione level significantly 
$(\mathrm{P}<0.05)$ decrease in non-supplemented diabetic subjects compared to non-diabetic and diabetic supplemented with Alphabetic subjects (Table 1), after supplementation with Alphabetic, the reduced glutathione levels return almost close to non-diabetic subjects (Table 1). Reduced glutathione detoxifies reactive oxygen radicals by giving them an electron which effectively neutralizes them, also reduced glutathione biotransforms hydrogen peroxide by turning it into harmless water [30]. Secondly, reduced glutathione recycles oxidized lipoic acid, vitamin C and E by restoring them to an active state, mostly by donating the electrons that they used in metabolizing (neutralizing) free radicals [31]. So, instead of having this army of antioxidants flushed out, they are recycled by reduced glutathione and sent back out to work.

Serum concentration of malondialdehyde (MDA), a marker of lipid peroxidation was significantly $(\mathrm{P}<0.05)$ higher in non-supplemented diabetic subjects compared to non-diabetic and supplemented diabetic subjects (Table 1), this suggests that the natural antioxidant defense mechanism to scavenge excessive free radical has been depleted in nonsupplemented diabetic subjects. Decrease in antioxidant enzyme activity as well as increased MDA as observed in diabetes mellitus might be due to an altered intracellular ratio between free radicals and antioxidant capacity because the reactive oxygen species (ROS), which are excessively produced in diabetes, are able to overwhelm the endogenous defense systems leading to oxidative stress [32][12]. ROS are considered important independent risk factors developed in diabetes mellitus via what is known as "auto-oxidative glycosylation", a process which is relevant at elevated blood glucose level. Hyperglycemia may also raise aldose reductase activity which depletes NADPH cell stores, thus perturbing the defense system [33][34]. The elevated blood glucose level can also cause non-enzymatic glycation of plasma proteins leading to the production of more powerful oxidizing species which can bind with most normal cellular components to "pair up" its unpaired electrons; thus, they react with the unsaturated bonds of membrane lipids, denature the proteins, and attack nucleic acids, resulting in cellular oxidative damage [35].

Serum concentration of antioxidant vitamin $\mathrm{A}$ and $\mathrm{C}$ were significantly $(\mathrm{P}<0.05)$ low in non-supplemented diabetic subjects compared with supplemented diabetic subjects (Table 2). This finding are in agreement with that of [35], who reported significantly $(\mathrm{P}<0.05)$ low level of antioxidant vitamins in diabetes. Some studies indicated that this deficiency is correlated with presence of diabetic complication [36].

Hyperglycemia generates reactive oxygen specie (ROS) and also inhibits antioxidant mechanism, creating a state of oxidative stress. The $\beta$-cells are sensitive to oxidative stress because their cellular antioxidant defence mechanism are weak [37]. It was also observe that there was an increase concentration of the vitamins in treated diabetic subjects ( $\mathrm{P}<$ 0.05). This may be due to supplementation with Alphabetic which is a good source of vitamin $\mathrm{C}$ and $\mathrm{E}$ which acts as scavengers of free radicals generated by glycosylated proteins [38]. Vitamin C cooperates with Vitamin E to regenerate $\alpha$ tocopherol from $\alpha$-tocopherol radicals in membranes and lipoproteins, and also raises intracellular glutathione levels thus playing an important role in protein thiol group protection against oxidation [39]. Ascorbic acid is a reducing agent and can reduce and thereby neutralize ROS such as hydrogen peroxide [39]. During the antioxidant reaction, $\alpha$-tocopherol is converted to an $\alpha$-tocopherol radical by the donation of a labile hydrogen to a lipid or lipid peroxyl radical, and the $\alpha$ tocopherol radical can therefore be reduced to the original $\alpha$-tocopherol form by ascorbic acid [40]. The main function of Vitamin E is to protect against lipid peroxidation [40]. Vitamin E lowers LDL-oxidation, thus lowering the risk of diabetic cardiovascular complications [41]. A study conducted by [42], shows that the nutrients including Vitamin E, Vitamin C, Magnesium, Chromium, Zinc and Manganase all have beneficial effects on symptoms or complications associated with diabetes. Many of these nutrients appear to be closely associated with insulin metabolism and help maintain proper blood glucose level. A serum level of $\beta$-carotene shows significant $(\mathrm{P}<0.05)$ increase in supplemented diabetic subject compared to non- diabetic subjects and also there is significant $(\mathrm{P}<0.05)$ increase in non-diabetic subjects compared to diabetic nonsupplemented subjects (Table 2). These possibly happen because $\beta$-carotene has the ability to protect the body against the destructive effects of free radicals and neutralize them by donating one of their own electrons, ending the electron"stealing" reaction [43]. $\beta$-carotene also act as scavengers, helping to prevent cell and tissue damage that could lead to cardiovascular disease and cancer [33]. There is a report that $\beta$-carotene has the ability to prevent oxidation of lowdensity lipoprotein (LDL) cholesterol and its harmful effects i.e development of fatty build-ups in the arteries (atherosclerosis) [29].

The result of correlation of serum glucose with serum antioxidant vitamins and oxidative stress markers of diabetics-supplemented and diabetics non- supplemented subjects (Table 3). The result shows that all the serum antioxidant vitamins and reduced glutathione are negatively correlated with serum glucose levels of both the supplemented diabetic subjects and non-supplemented diabetic subjects. Oxidative stress marker (MDA) shows a positive correlation with serum glucose of both diabetic non- supplemented and diabetic supplemented. Increased oxidative stress in diabetic subjects results in higher utilization of these minerals and vitamins and consequently their deficiencies. After supplementation with Alphabetic which is good source of antioxidants minerals and vitamins almost their levels return to normal. Also the results revealed that, as the level of glucose increase than the levels of antioxidant vitamins and reduced glutathione will also decrease while as the level of glucose increases then the level of oxidative stress marker (MDA) will also increases (Table $3)$. 
This findings are in agreement with the findings of West [34], who reported Correlation of Serum Glucose with Serum Antioxidant Vitamins and Trace Elements of Diabetics in Sokoto and finds that all the serum antioxidant vitamins and trace elements are negatively correlated $(\mathrm{P}<0.05)$ with serum glucose levels of the diabetics.

\section{CONCLUSION}

The current work reported that supplementation of diabetic subjects with antioxidant rich supplement (Alphabetic) which are known source of multivitamin, Mineral elements, alpha lipoic acid e.t.c, significantly may reverse oxidative stress of diabetic subjects. The results indicated that the supplementation reduces serum level of MDA and FBS then increase serum level of GSH, Vitamin E, Vitamin C and $\beta$-carotene.

Based of these findings, it can be concluded that supplementation with antioxidants rich supplement (Alphabetic) may reverse the risk of oxidative stress associated with diabetes leading to diabetic complications.

\section{ACKNOWLEDGEMENT}

The authors want to thank the Usmanu Danfodiyo University and Usmanu Danfodiyo University Teaching Hospital for their Support.

\section{REFERENCES}

[1] Saidu, Y., Nwachukwu, F.C., Bilbis, L.S., Faruk, U.Z. and Abbas, A.Y. (2010). Hypoglycaemic and Hypolipidemic Effects of Root Extracts of Albizzia chevalieri in Alloxan-induced Diabetic Rats. Nig J of Basic and Applied Sci. 18(1): $72-78$.

[2] Ladan, M.J., Umar R.A., Hassan S.W., Shehu B. (2007). Glycemic Status and Lipid Profile of Diabetes in Sokoto, Nigeria. Asian J of Biochem. 2: 183- 187

[3] ADA (American Diabetes Association). (2012). "Diagnosis and classification of diabetes mellitus," Diabetes Care, 35(1). Pp. 64-71. Antioxidants: A review. J. of Biochem and Mol. Tox., 17(1): 24.

[4] Cade, W. T. (2008)“Diabetes-related microvascular and macrovascular diseases in the physical therapy setting," Phy. Ther., 88(11): 1322-1335.

[5] Whiting, D.R., Guariguata, L., Wei,l C. and Shaw, J. (2011). IDF Diabetes Atlas: global estimates of the prevalence of diabetes for 2011 and 2030. Diabetes Res Clin Pract. 94:311-321.

[6] Shaw, J.E. , Sicree, R.A., Zimmet, P.Z. (2010). Global estimates of the prevalence of diabetes for 2010 and 2030. Diabetes Res. Clin. Pract. 87: 4-14.

[7] WHO (World Health Organization) Report (2011). Prevalence of Diabetes Mellitus. Retrieved on 2012-11-10-11.

[8] Wild, S., Roglic, G., Green, A., Sicree, R. and King, H. (2004). Global prevalence of diabetes: estimates for the year 2000 and projections for 2030. Dia. Car., 27: 1047-1053.

[9] Leduc, C., Coonishish, J., Haddad, P. and Cuerrier, A. (2006). Plants used by the Cree Nation of Eeyou Istchee (Quebec, Canada) for the treatment of diabetes: a novel approach in quantitative ethnobotany. J Ethnopharmacol. 105: 5563.

[10] Amos, A.F., McCarty, D.J. and Zimmet, P. (1997). The Rising Global Burden of Diabetes and its complication: Estimates and Projections to the year 2010. Dia. Med. 14:81-85.

[11] Chase, H.P., Jackson, W.E., Hoops, S.L., Cockerham, R.S., Archer, P.G. and O’Brien, D. (1989). Glucose control and the renal and retinal complications of insulin-dependent diabetes. JAMA. 261:1155-1160.

[12] Bilbis, L. S. (2008). 'Friends or Foes' Reactive oxygen species in health and diseases. Usmanu Danfodiyo University Central University Serminar Series, Augues $28^{\text {th }} 2008$. Pp. 14

[13] Chong, Z.Z., Li, F. and Maiese, K. (2005). Oxidative stress in the brain: Novel cellular targets that govern survival during neurodegenerative disease. Prog Neurobiol., 75: 207-246. 
[14] Ha, H. and Lee, H.B. (2000). Reactive oxygen species as glucose signaling molecules in mesangial cells cultured under high glucose. Kidney Int., 77: 19-25.

[15] Maritim, A.C., Sanders, R.A. and Watkins, J.B. (2003). Diabetes, oxidative stress, and antioxidants: a review. J Biochem Mol Toxico.l 17: 24-38

[16] Rains, J. L., \& Jain, S. K. (2011). Oxidative stress, insulin signaling, and diabetes. Free Radic Biol Med. 50(5): 567575.

[17] Daniel, W. W. W. (1999). Biostatistics: a foundation for analysis in the health science. $7^{\text {th }}$ Ed. New York, NY: Wiley. 180- 185, 268- 270.

[18] Trinder, P. (1969). Determination of blood glucose in blood using glucose oxidase with an alternative oxygen acceptor, Annals of Clin Biochem, 6:24-25.

[19] Pett, L. B., (1940). Fat soluble vitamins. Ann. Rev. of Biochem. 14: 525-560.

[20] Baker, H. and Frank, O. (1986). Determination of serum tocopherol. In: clinical biochemistry, $6^{\text {th }}$ edition. Eimememenn medical books, London, Pp; 902-903.

[21] Hashim S. A. and Schuttringer G. R. (1966). Rapid determination of tocopherol inmacro- and microquantities of plasma. Results obtained in various nutrition and metabolic studies. Am. J. Clin. Nutri; 19(2):137- 14.

[22] Patterson, J.W., Lazarow, A. (1959). Sulfhydryl protection against dehydroascorbic acid diabetes. J. Biol Chem., 186: 141-144.

[23] Shah, J. K. and Walker, A. M. (1989). Quantitative Determination of Malondialdehyde, Biochem. Biopy. Acta. 15 (11): 207-211.

[24] Asayama, K., Uchida, N., Nwakele, T., Hayashibe, H. and Dobashi, K., (1993). Antioxidant in serum of children with type 1 DM. Free rad. Bio med. 15(6):597-602

[25] Zingg, J. M., Ricciarelli, R. and Azzi, A. (2000). Scavenger receptors and modified lipoproteins. Fatal attraction. IUBMB Life. 49:397-403.

[26] Paolisso, G. and Giugliano, D. (1996). Oxidative stress and insulin action: is there a relationship? Diabetologia. 39:357-36

[27] Kalaivanam, K.N. Mala, D., Sara, R. (2006). Lipid peroxidation in type 11 diabetes mellitus. I J of dia in develop coun. 26:30-32

[28] Fridivich, I. (1995). Superoxide radical and superoxide dismutase. Ann rev biochem. 64:97-112.

[29] George, O. (2009). Antioxidants properties of Vitamins. J of American Fam phy. 60: 895-904

[30] Mates, J.M. and Sanchez-Jimenez, F.M. (1999). Antioxidant enzymes and their implications in pathophysiologic processes. Front Biosci. 15: 339-345

[31] Satyanarayana, U., and Chakrapani, U. (2006). Biochemistry. Books and Allied (P) Ltd. Third edition Pp: 409-412.

[32] Sarita, A. Shinde, Anita, D. Deshmukh, Adinath, N. Suryakar, Umesh, K. More, Mona, A. Tilak. (2014). The levels of oxidative stress and antioxidants in diabetes mellitus before and after diabetic treatment with or without antioxidants. Indian J of Basic and App Med Res. 3(2): 455-460.

[33] Britton, K., Baynes, J.W., Peter, U. (1995). Role of oxidative stress in development of complication of diabetes. $J$ of Dia. 40: 305-320

[34] West, I. C. (2000). Radicals and oxidative stress in diabetes. Diabet Med. 17:171- 180.

[35] Aliyu, M., Lawal, M., Mojiminiyi, F., Saidu, Y. and Bilbis, L.S. (2005). Serum antioxidant vitamin levels in in Noninsulin dependant diabetes mellitus subject in Sokoto, Nigeria. J Nig. Soc. For exper. biol.17(2): 107-114 
[36] Moorodian, A.D. and Morley, B. (1987). Narrative review a rational approch to stating insulin therapy. Ann Inter med. 145(2): 125-134.

[37] Rasilainens, K. Kuther, S.R. and Peter U.K. (2002). Type ll DM. J dia. 82:201-212

[38] Polidori, M. C. (2005). Plasma level of lipophilic antioxidants in every old patients with type ll diabetes. Dia met res rev. 16:15-19

[39] Broekmans,W.M.R., Klopping-Ketelaars, C.R.W.C and Schuurman (2000). Fruits and vegetable increase plasma carotenoids and Vitamins reduce homocysteine in human. J of Nutri 130(6): 1578-1583.

[40] Pryor W.A. (1996). Vitamin E and Carotenoid Abstracts. 1994 Studies of Lipid-Soluble Antioxidants. Vim. E Resrch and Inform. Serv.publishers.

[41] Fuller, C. J. Chandalia, M. and Garg, A. (1996). Alpha tocopheryl acetate supplementation at pharmacological doses decrease low-density lipoprotein oxidative susceptibility but not protein glycation in patients with diabetes mellitus. A J clinical nitri., 63: 753-759

[42] Chung, A. M., Yu, J.J. and Liu, D.Z. (1998). Alpha tocopherol reduces lipid peroxidation stress of erythrocyte membrane in type 11 diabetes mellitus patients with retinopathy. Dia., 40: 380-385

[43] Terry, C. (2005). Diabetes Mullitus. J of clin nutri. 82: 3685-3698

[44] Wali,U., M.U Jogana, A.L Zarummai and Y. Saidu. (2011). Antioxidant Vitamins and Trace Elements Status of Diabetics in Sokoto, Nigeria. Nig J of Basic and App Sci. 19(1):130- 134. 